\title{
Fault Diagnosis for Power Transformer Based on SVM Information Fusion
}

\author{
Sima Li-ping ${ }^{1, \text { a }}$, Su Xing-zhi' ${ }^{2, b}$, Wang Bo ${ }^{1, \mathrm{c}}$, Dou Peng ${ }^{2, \mathrm{~d}}$, Liu Gen-cai ${ }^{2, \mathrm{e}}$ and \\ Shu Nai-qiu ${ }^{1, f}$ \\ ${ }^{1}$ School of Electrical Engineering, Wuhan University, Wuhan, China \\ ${ }^{2}$ Foshan Power Supply Bureau, Guangdong Power Grid Corporation, Foshan, China \\ asmlping@163.com, b13902800139@139.com, cwhwdwb@gmail.com, ddoupeng0106@163.com, \\ eliugenial@163.com, 'shunaiqiu1@163.com
}

Keywords: transformer; SVM; information fusion; fault diagnosis

\begin{abstract}
A kind of fault diagnosis method for power transformer based on multi-SVM information fusion is proposed in this paper. Based on the "one against one" multi-classification SVM and pairwise coupling method, the multi-classification SVM probability outputs are obtained and regarded as the basic probability function of information fusion to diagnose. It also makes full use of complementary information from gases dissolved in transformer oil analysis data and electrical test data. From the example analysis results, it shows better performance than a single feature SVM method in the aspect of accuracy and generalization.
\end{abstract}

\section{Introduction}

Power transformer is the important power distribution equipment of power system. Transformer fault diagnosis has become one of the hotspots which the electric power industry concern with. Recently, the method based on the principle of data fusion, which makes use of the dissolved gas data and electrical test data, gradually spread. It has obtained some achievements ${ }^{[1]}$. But in practical applications, theory of evidence has not given the specific construction method of basic probability distribution, and the credible degree of the evidence is also difficult to determine. It is a kind of very good trial that using machine learning algorithm based on fault samples distributes $\mathrm{BPA}^{[2]}$. Literature [3] has established BPA by using neural network, but this method also has some disadvantages, for example, lack of fault sample, network structure hard to determine, slow convergence speed and local minimum values, over learning and owe learning etc.

To solve these problems above, a transformer fault diagnosis method combined SVM with information fusion organically is proposed. It uses the "one against one" multiclass support vector machine to distribute BPA. So it objectifies the basic probability assignment, and avoids the complexity of basic probability distribution function constructed in information fusion.

\section{Relevant Theory}

\section{A. Posterior probability estimate based on Support Vector Machines}

Support Vector Machines put forward by Vapnik is a new general method of machine learning, which is based on statistical learning theory ${ }^{[4]}$. Wahba and Platt introduced the posteriori probability into standard support vector machine (SVM) in $2000^{[5]}$. The support vector machine of posterior probability, proposed by Platt, has achieved good effect in the actual application. However, this method can only handle the problem of two classifications. For the probability estimate of multi classification problem, at present it is the main method that getting the posterior probability of sample in case of multi classification by optimizing probability results of many two classifications ${ }^{[6]}$. This paper use the Pairwise Coupling ${ }^{[7]}$, put forward by Hastie, and "one against one" support vector machine multi classification method to finish that probability paired off transforms into multi classification probability. We can achieve all kinds of posterior probability value $p_{i}=p(y=i \mid x)$ by solving the following optimal problem: 


$$
\left\{\begin{array}{l}
\min _{p} \frac{1}{2} \sum_{i=1}^{k} \sum_{j: j \neq i}\left(r_{j i} p_{i}-r_{i j} p_{j}\right)^{2} \\
\sum_{i=1}^{k} p_{i}=1, \quad p_{i} \geq 0, \forall i,
\end{array}\right.
$$

In the expression, $r_{i j}=p(y=i \mid y=i$ or $j, x)$, Here, $i, j=1,2, \ldots, k$, and $i \neq j, \quad x$ belongs to the posterior probability of class $i$, when class $i$ and class $j$ are paired off.

\section{B. The evidence theory}

The essence of Dempster-Shafer data fusion theory is the process which synthesizes the different evidence to new evidence in the same recognition framework by using the evidence combination rules. Three elements of evidence theory are: basic probability assignment $m(A)$, belief function $\operatorname{Bel}(A)$ and plausibility function $\operatorname{Pl}(A)$. $[\operatorname{Bel}(A), P l(A)]$ constitutes a confidence interval that determine the bound of the current evidence to the belief function of $\mathrm{A}$.

Suppose that $\alpha=A_{i_{1}} \cap A_{2 i_{2}} \cap, \ldots, \cap A_{i_{i}}, K_{1}=\sum_{\alpha=\phi} f_{1}\left(A_{i_{1}}\right) f_{2}\left(A_{2 i_{i}}\right) \ldots f_{t}\left(A_{i_{i}}\right)$, then fused belief function is:

$$
m(A)=\left\{\begin{array}{c}
\frac{\sum_{\alpha} m_{1}\left(A_{i_{1}}\right) m_{2}\left(A_{2 i_{2}}\right) \cdots m_{t}\left(A_{i_{i}}\right)}{1-\sum_{\alpha=\phi} m_{1}\left(A_{i_{1}}\right) m_{2}\left(A_{2 i_{i}}\right) \cdots m_{t}\left(A_{i_{i}}\right)} \quad \alpha \neq \phi \\
0 \quad \alpha=\phi
\end{array}\right.
$$

\section{Transformer Fault Diagnosis Based on SVM Information Fusion}

\section{A. Definition of fault space}

Through statistical analysis of many transformers' fault position and relevant documents, this paper determine the fault space in consideration of transformer internal structure. the fault space : \{P1 winding, P2 tap changing switch and lead, P3 iron core, P4 structural components and Magnetic shield, P5 insulating barriers and stay, P6 floating potential body, P7 transformer oil \}.

\section{B. Definition of fault symptom space}

Different characteristic signal reflect the real situations of the transformer faults in different aspects. Considering the various trials conditions, according to collect the fault samples, this paper determines the fault omen space:\{DGA, iron core grounded current and iron core insulation resistance, tri-phase imbalance rate of winding direct current resistance, deviation of winding ratio, absorption and polarization index of winding, Moisture content in oil\}.

\section{Construct of evidences}

Combining with proposition characteristics and fault omen space in the recognition framework, this paper constructs two SVMs to process DGA and electrical test data respectively, and takes the output of each SVM as independent evidence. The diagnosis model shows in figure 1.

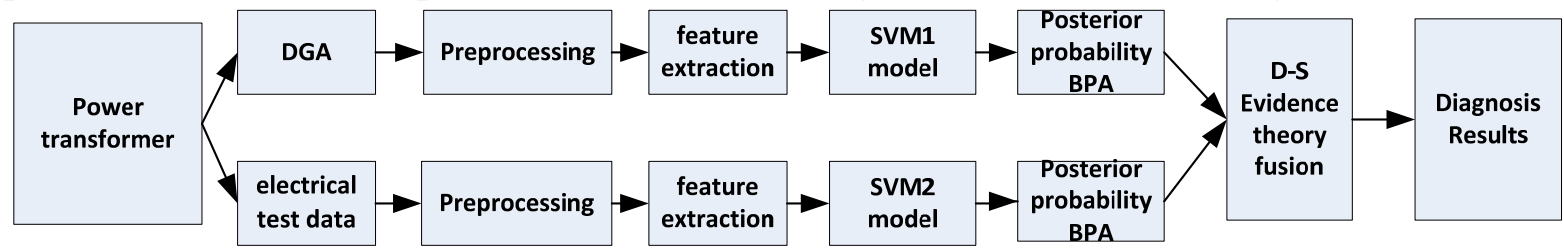

Fig. 1 Transformer fault diagnosis framework based on SVM information fusion

Five ratios $\mathrm{C}_{2} \mathrm{H}_{2} / \mathrm{C}_{2} \mathrm{H}_{4}, \mathrm{CH}_{4} / \mathrm{H}_{2}, \mathrm{C}_{2} \mathrm{H}_{4} / \mathrm{C}_{2} \mathrm{H}_{6}, \mathrm{C}_{2} \mathrm{H}_{6} / \mathrm{CH}_{4}, \mathrm{CO} / \mathrm{CO}_{2}$ is used as the input parameter to construct the evidence of SVM1. And electrical test data is used as parameter space in SVM2.

\section{The BPA of assignment by SVM}

In the application of the evidence theory, it is very important to achieve the basic probability assignment function. This paper uses the probability output of SVM as BPA, and obtains the accuracy of corresponding classification of evidence by SVM's training. It makes the basic probability distribution assignment objective, and solves assignment problem of belief function in information fusion effectively. In the system, seven types fault are recognized by evidence fusion of 
two multi classification SVMs. Its recognition framework is $\Theta=\left\{P_{1}, P_{2}, \ldots P_{7}\right\}$, and belief function assignment of each proposition about evidence $\mathrm{j}$ is:

$$
\left\{\begin{array}{c}
m_{j}\left(P_{1}, P_{2}, \ldots P_{7}\right)=\left(r_{j} p_{j 1}, r_{j} p_{j 2}, \ldots, r_{j} p_{j 7}\right) \\
m_{j}(\Theta)=1-r_{j}, j=1,2
\end{array}\right.
$$

Here, $p_{j 1}, p_{j 2}, \ldots, p_{j 7}$ - the posteriori probability calculation value when the evidence $\mathrm{j}$ of multi classification $\mathrm{SVM}$ is judged as $P_{1}, P_{2}, \ldots P_{7}$.

$r_{j}$ — the accuracy rate of the evidence $\mathrm{j}$ of multi classification SVM.

$m_{j}\left(P_{1}, P_{2}, \ldots P_{7}\right)$ — the belief function assignment of the evidence $\mathrm{j}$ of multi classification SVM.

$m_{j}(\Theta)$ _ uncertainty belief function assignment of the evidence $\mathrm{j}$ of multi classification SVM.

We suppose the probability that an unknown class examples can be correctly identified is $r_{j}$, so the probability that SVM classifier fails to identify an example is $1-r_{j}$, Then in basic probability assignment, we give the wrong classification ratio $1-r_{j}$, to the recognition framework $m_{j}(\Theta)$.

\section{E. Combination evidence and Making diagnosis}

By using certain BPA, according to the definition of evidence theory, we can calculate belief function and plausibility function of each proposition. With regard to belief function of different evidence, by using the expression (2), to calculate belief function and plausibility function in the combining of each evidence, and to get the confidence interval, $\left[B \mathrm{Bl}_{i}, P l_{i}\right]\left[B e l_{i}, P l_{i}\right]$, of all propositions in recognition framework $\Theta$ about evidence, and the uncertainty, $m_{j}(\Theta)$, of evidence. Then in this paper we conclude decision-making rules as follow:1) The belief function of target fault is the maximum in all kinds of belief function. That is $\left.\operatorname{Bel}\left(A_{1}\right)=\max \left\{\operatorname{Bel}\left(A_{i}\right), A_{i} \subset \Theta\right\} ; 2\right)$ Between the belief function of target fault and the other faults, the difference in value should be more than a certain threshold $\varepsilon_{1}$. That is $\left.\operatorname{Bel}\left(A_{1}\right)-\operatorname{Bel}\left(A_{2}\right)>\varepsilon_{1}, \operatorname{Bel}\left(A_{2}\right)=\max \left\{\operatorname{Bel}\left(A_{1}\right), A_{1} \subset \Theta, A_{1} \neq A_{1}\right\} ; 3\right)$ uncertainty belief function should be under a certain threshold $\varepsilon_{2}$. That is $m(\Theta)<\varepsilon_{2}$.According to reality, we determine some settings, $\varepsilon_{1}$ equals to 0.4 and $\varepsilon_{2}$ equals to 0.36 .

\section{Fault Diagnosis Example}

A main transformer typed SFPS9-150000/220, in August 2008 after the thunderstorm, the total hydrocarbon of dissolved gases in transformer oil is abnormal, and acetylene exceeds badly, shown in table 1 . In the winding DC resistance test, the imbalance rate of medium voltage side phase B of straight resistance is $2.23 \%$, the deviation of transformer ratio test is as high as $21.83 \%$, Insulation resistance test is qualified, but polarization index is as low as 1.33 ,the others are qualified.

Table 1. The DGA data of a main transformer $[\mathrm{ul} / \mathrm{L}]$

\begin{tabular}{|c|c|c|c|c|c|c|c|}
\hline Time & $\mathrm{H}_{2}$ & $\mathrm{CH}_{4}$ & $\mathrm{C}_{2} \mathrm{H}_{6}$ & $\mathrm{C}_{2} \mathrm{H}_{4}$ & $\mathrm{C}_{2} \mathrm{H}_{2}$ & $\mathrm{CO}$ & $\mathrm{CO}_{2}$ \\
\hline 2008.8 .26 & 168 & 42.7 & 5.1 & 50.3 & 55.4 & 339 & 2756 \\
\hline
\end{tabular}

After the pretreatment of data above in fuzzy, the output calculated by SVM1 2 probability estimate model is shown in the table 2. Here, $r_{j}$ stands for the accuracy rate of evidence classification of each SVM.

Table 2. Probability output and accuracy rate of SVM1 2

\begin{tabular}{|c|c|c|c|c|c|c|c|c|}
\hline Evidence & $r j$ & $P 1$ & $P 2$ & $P 3$ & $P 4$ & $P 5$ & $P 6$ & $P 7$ \\
\hline SVM1 & 0.6581 & 0.2402 & 0.2148 & 0.1319 & 0.0744 & 0.1573 & 0.1254 & 0.0560 \\
\hline SVM2 & 0.8376 & 0.8182 & 0.0728 & 0.0283 & 0.0424 & 0.0130 & 0.0178 & 0.0075 \\
\hline
\end{tabular}

Table 3 shows that a diagnosis result of single characteristic SVM and SVM information fusion. The recognition result of SVM1 2 fused is winding fault. Actually, when we took away the cover, we found the winding deformative obviously, and metallicity Turn-to-turn Short Circuit exists in the medium voltage side of the winding. The diagnosis conclusions and de inspection results were corresponding. 
Table 3. Single action of each evidence, confidence interval after fusing $[\operatorname{Bel}(A), P l(A)]$ and diagnosis results

\begin{tabular}{|c|c|c|c|c|c|c|c|c|c|}
\hline Evidence & $\operatorname{mi}(\Theta)$ & $P 1$ & $P 2$ & P3 & $P 4$ & $P 5$ & $P 6$ & P7 & Results \\
\hline \multirow[t]{2}{*}{ SVM1 } & \multirow[t]{2}{*}{0.3419} & 0.1581 & 0.1414 & 0.0868 & 0.0490 & 0.1035 & 0.0825 & 0.0369 & \multirow[t]{2}{*}{ Not sure } \\
\hline & & 0.5000 & 0.4833 & 0.4287 & 0.3909 & 0.4454 & 0.4244 & 0.3788 & \\
\hline \multirow[t]{2}{*}{ SVM2 } & \multirow[t]{2}{*}{0.1624} & 0.6853 & 0.0610 & 0.0237 & 0.0355 & 0.0109 & 0.0149 & 0.0063 & \multirow[t]{2}{*}{$\mathrm{P} 1$} \\
\hline & & 0.8477 & 0.2234 & 0.1861 & 0.1979 & 0.1733 & 0.1773 & 0.1687 & \\
\hline \multirow{2}{*}{$\begin{array}{l}\text { SVM1 2 } \\
\text { After fusing }\end{array}$} & \multirow{2}{*}{0.0528} & 0.6092 & 0.1133 & 0.0443 & 0.0312 & 0.0568 & 0.0787 & 0.0135 & \multirow{2}{*}{$\mathrm{P} 1$} \\
\hline & & 0.6621 & 0.1661 & 0.0971 & 0.0841 & 0.1097 & 0.1316 & 0.0664 & \\
\hline
\end{tabular}

As shown in Table 3, when the actual fault is P1 (winding fault), the P1 confidence interval about the evidences SVM1 and SVM2 are [0.1581,0.5000] and [0.6859,0.8477], their uncertainty are 0.3419 and 0.1624 . According to the fused diagnosis decision-making rules, only the evidence SVM2 can reach the diagnosis conclusion P1. After fusing of two evidences the confidence interval is [0.6092,0.6621], uncertainty decreases to 0.0528. Obviously belief function improves, and diagnosis conclusion is right.

\section{Conclusions}

This paper proposes a transformer fault diagnosis method based on multi SVM information fusion. Then characteristics of the method are as follow:1)By using the pairwise coupling algorithm and introducing probability estimate model in multi classification SVM, this method overcomes the traditional SVMs' defect that the hard output limits later processing in multi classification problem. And it solves the probability output problem of SVM effectively; 2)By taking the probability output as the basic probability assignment in information fusion recognition framework, this method makesBPA objective, and avoid the complexity of constructing BPA in information fusion; 3)By making full use of the complementary information of DGA and electrical test data, this paper combines the SVM with evidence theory to diagnose the position where the transformer fault could exist. Compared with departed diagnosis based on single characteristic transformer fault, this method is more significant on deciding the transformer maintenance strategy.

\section{References}

[1] Xiong Hao,Yang Jun,Li Wei-Guo,etc. Application of Evidence Theory to Power Transformer Faults Synthesized Diagnosis[J]. Proceedings of the CSEE. 2008, 28(28): 24-30.

[2] Jiang Wan-lu,Wu Sheng-qiang. Multi-data Fusion Fault Diagnosis Method Based on SVM and Evidence Theory[J]. Chinese Journal of Scientific Instrument. 2010, 31(8): 1738-1743.

[3] Liao Rui Ji,Liao Yu Xian,Yang Li Ju,etc. Study on Synthetic Diagnosis Method of Transformer Fault Using Multi-neural Network and Evidence Theory[J]. Proceedings of the CSEE. 2006, 26(3): 119-124.

[4] Vapnik. V N. The Nature of Statistical Learning Theory[M]. New York: Springer-Verlag, 1995.

[5] Platt J C. Probabilistic Outputs for Support Vector Machines and Comparison to Regularized Plausibility function Methods.[M]. Advances in Large Margin Classifiers, A. Smola P B B S, Cambridge, MA, 2000. MIT Press.

[6] Zhang Xiang,Xiao Xiao-ling,XU Guang-you. Weighted posterior probability output for support vector machines[J]. Journal of Tsinghua University(Science and Technology). 2007, 47(10): 1689-169.

[7] Hastie T T R. Classification by Pairwise Coupling[J]. The Annals of Statistics. 1998, 26(1): 451-471. 